\title{
Quantitative study of the transmission of axially channeled protons in thin silicon crystals
}

\section{Citation}

Rosner, J. S., W. M. Gibson, J. A. Golovchenko, A. N. Goland, and H. E. Wegner. 1978. Quantitative Study of the Transmission of Axially Channeled Protons in Thin Silicon Crystals. Physical Review B. doi:10.1103/physrevb.18.1066.

\section{Published Version}

doi:10.1103/PhysRevB.18.1066

\section{Permanent link}

http://nrs.harvard.edu/urn-3:HUL.InstRepos:34334608

\section{Terms of Use}

This article was downloaded from Harvard University's DASH repository, and is made available under the terms and conditions applicable to Other Posted Material, as set forth at http:// nrs.harvard.edu/urn-3:HUL.InstRepos:dash.current.terms-of-use\#LAA

\section{Share Your Story}

The Harvard community has made this article openly available.

Please share how this access benefits you. Submit a story.

\section{Accessibility}




\title{
Quantitative study of the transmission of axially channeled protons in thin silicon crystals
}

\author{
J. S. Rosner* \\ Rutgers University, New Brunswick, New Jersey 08903 \\ W. M. Gibson ${ }^{\dagger}$ and J. A. Golovchenko \\ Bell Laboratories, Murray Hill, New Jersey 07974
}

\author{
A. N. Goland and H. E. Wegner \\ Brookhaven National Laboratory, Upton, New York 11973 \\ (Received 23 March 1978)
}

\begin{abstract}
The azimuthal distributions of protons transmitted through thin silicon single crystals near the $\langle 110\rangle$ axis were measured using a two-dimensional position-sensitive detector. The data are composed of ringlike distributions with strong azimuthal and transverse energy dependence. The azimuthal distributions are compared with theoretical predictions based on the random string approximation using different forms of the interatomic potential. "Blocking" in the transverse plane is also observed. In addition, from an analysis of the radial spreading of the distribution the effects of inelastic scattering in the transverse plane are clearly seen.
\end{abstract}

\section{INTRODUCTION}

The experimental study of particle-channeling effects in crystalline material has been vigorously pursued for over 13 years, and the general concepts required to obtain an overall comprehension of the subject are reasonably well established. Nevertheless, several aspects of the channeling effect still seem worthy of study, either from the standpoint of providing detailed quantitative results of particular use in application, or with the idea of critically examining from different perspectives certain assumptions upon which the theory is built.

One may classify channeling experiments into two categories which, in principle, are capable of supplying complementary information about the channeling process. The first category consists of those experiments whose results are sensitive to the transverse position distribution of penetrating particles. Characteristic of this case are wide-angle Rutherford scattering experiments, where the scattering yield as a function of incident tilt angle of the beam to the crystal is measured.

In the second type of experiment with which this paper deals in detail, the distinguishing feature is that results are determined by the transversemomentum distribution. Transmission-type experiments, where the incident particles traverse a thin crystal and emerge from the back, characterize this case, as we shall see. Thus, the particular distribution observed, i.e., position or momentum, may be used to distinguish the two cases.

A number of years ago, a series of channeling experiments was performed to investigate the transmission of ions through thin single crystals. ${ }^{1}$ The angular distributions of transmitted particles were recorded using photographic film placed approximately $1 \mathrm{~m}$ behind the crystal. When the angle $\psi$, defined as the angle between a major axial direction and the incident beam, was less than the critical angle for channeling $\psi_{1}$ (Ref. 2) ringlike patterns were observed. These rings were found to be nonuniform. In all cases there was a modulation in intensity as a function of azimuthal angle which reflected the symmetry of the crystal. This was explained as "blocking" in the transverse plane. Blocking is defined as the restriction of particle motion from low-index axial (or planar) directions, arising from the extraordinary correlated scatterings from successive target atoms. In addition, as $\psi$ approached the critical angle, the intensity was increasingly peaked in the original beam direction.

The recent development of two-dimensional position-sensitive detectors, ${ }^{3}$ and the fabrication of thin uniform single crystals of silicon ${ }^{4}$ have made it possible for us to make a quantitative investigation of these ringlike distributions. A series of transmission experiments using the Brookhaven Research Van de Graaff accelerator was performed utilizing a beam of $3.2-\mathrm{MeV}$ protons, which were channeled along the $\langle 110\rangle$ axis in various thin silicon crystals. When the angle between the incident beam and the crystal axis was of the order of the critical angle, the characteristic ringlike intensity distribution was observed. This distribution will be referred to as a "doughnut." It will be shown that the azimuthal distribution of the doughnut depends on the form of the interatomic potential. 


\section{THEORY}

Figure 1 illustrates the geometry of the doughnut formation. As noted, for angles of incidence of the order of $\psi_{1}$, circular distributions are obtained on a screen or detector placed behind the crystal. The center of the circular region corresponds to the projection of the axial direction on the detector. The radius of the circular distribution is basically determined by the angle between the incident beam direction and crystal axis direction. For the purpose of interpreting these patterns within the framework of more standard channeling notions, we note that the distance from the axis projection at which a particle falls on the detector is determined by the projection of the emergent particle momentum perpendicular to the string direction. (The angles being dealt with in these experiments are so small, and the crystals so thin, that the momentum along the axis direction may be considered to be that of the incident beam and the effect of crystal tilt on this quantity may be neglected.)

Thus if we take the projection of the axis as the origin of our coordinate system located a distance $D$ from the crystal, a particle incident a distance $d$ from the origin has a transverse momentum of magnitude $(d / D) \sqrt{2 M E}$, where $E$ and $M$ are the projectile energy and mass, respectively. The azimuthal direction $\theta$ may be conveniently measured relative to the direction of the transverse momentum vector of the incident beam (Fig. 1). The resulting angular distribution of transmitted particles yields directly the transverse momentum distribution at the back surface of the crystal.

The basic shape of the azimuthal distribution has been theoretically treated using a randomstring approximation (RSA). ${ }^{5}$ This model assumes that the crystal is composed of strings of charge all aligned in the same direction, but distributed randomly in the transverse plane. The pair corre-

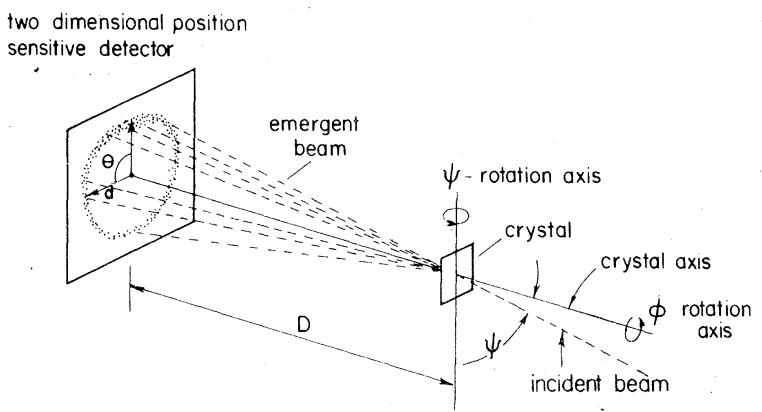

FIG. 1. Geometry of doughnut formation. Incident beam enters crystal at an angle $\psi$ with respect to the axis. As a result of collisions within the crystal, the direction of $p_{\perp}$ randomizes, yet remains almost constant in magnitude. The result is a ringlike distribution (a doughnut) when viewed on a phosphor screen or two-dimensional (2D) position-sensitive detector.

lation function $\xi(r)$, which is defined as the probability of a pair of strings being separated by a distance $r$, is taken to be equal unity, and the correlation between successive string collisions is neglected. The initial azimuthal distribution is taken to be $\delta(\theta)$. As the particles pass through the crystal, elastic collisions with the strings change the direction of the particle transverse momentum $p_{\perp}$. The time evolution of the direction of $p_{\perp}$ is then described by using the Boltzmann transport equation for the probability of finding a particle with transverse momentum in the direction $\theta$,

$\frac{\partial A(\theta, t)}{\partial t}=\int_{-\pi}^{\pi} d \theta^{\prime} P\left(\theta^{\prime}\right)\left[A\left(\theta-\theta^{\prime}, t\right)-A(\theta, t)\right]$,

where $P(\theta)$ is the transition rate obtained from the scattering potential and $A(\theta, t)$ is the probability of finding a particle at angle $\theta$ at time $t$. For potentials governing the collisions of the form $U(1 / r)$ $=c_{1} / r$ or $U\left(1 / r^{2}\right)=c_{2} / r^{2}$, exact analytic solutions to the transport equation have been found. ${ }^{5}$ The

TABLE I. Theoretical azimuthal distributions.

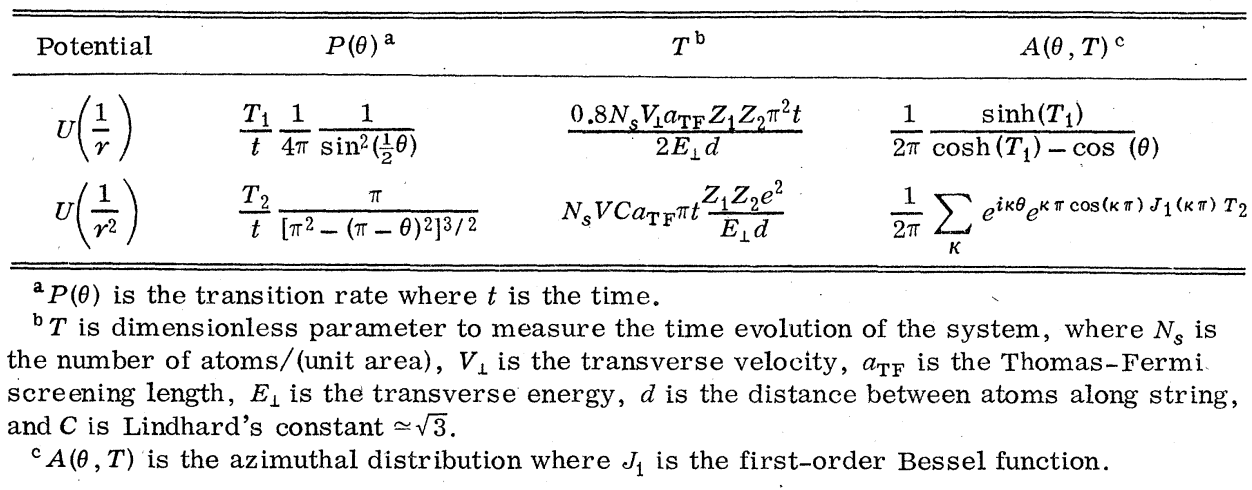


forms of the azimuthal distributions $A_{1 / r}(\theta, T)$ and $A_{1 / r_{2}}(\theta, T)$ are summarized in Table I. $T$ is a dimensionless measure of the time which depends on the scattering potential and the crystal thickness penetrated.

The constants $c_{1}$ and $c_{2}$ are chosen to be 0.8 $\times Z_{1} Z_{2} e^{2} \pi a_{\mathrm{TF}} / 2 d$ and $Z_{1} Z_{2} e^{2} C^{2} a_{\mathrm{TF}}^{2} / d$, respectively. The potential $U(1 / r)$ is the potential used by Lindhard to estimate the approach to statistical equilibrium in the transverse motion, ${ }^{6}$ and $U\left(1 / r^{2}\right)$ is an expansion of the Lindhard standard string potential $^{6}$

$$
U(\boldsymbol{r})=\left(Z_{1} Z_{2} e^{2} / d\right) \ln \left[1+\left(C a_{\mathrm{TF}} / r\right)^{2}\right]
$$

Justification for the choice of the constants $c_{1}$ and $c_{2}$ can be seen in Fig. 2. Here the model potentials are compared with the Molière approximation to the Thomas-Fermi potential. ${ }^{7}$ One can see that for $r<2 a_{\mathrm{TF}}$, the $U(1 / r)$ potential is in good agreement with the Thomas-Fermi potential. The factor 0.8 was introduced to give a best fit in the region $\mu_{1}<r<2 a_{\mathrm{TF}}$, where $\mu_{1}$ is the thermal vibration amplitude and $a_{\mathrm{TF}}$ is the Thomas-Fermi screening length. For $r>2 a_{\mathrm{TF}}$, the $U\left(1 / r^{2}\right)$ potential gives the best agreement with the ThomasFermi potential. For this case, no normalization adjustment is necessary.

\section{EXPERIMENT}

Since nearly all particles are transmitted through the thin $(1900-7500 \AA)$ crystals used in the experiment, an extremely low beam current was necessary $(\sim 100-200$ counts/sec $)$ to prevent electronic pulse pileup in the counting system. This was accomplished by detuning the accelerator and electrostatically deflecting the beam before two $0.5-\mathrm{mm}$ collimators spaced $2 \mathrm{~m}$ apart so that only a very tiny fraction of the beam was incident on the crystal.

The thin silicon crystals, prepared at Bell Laboratories, ${ }^{4}$ were produced by selective chemical etching of boron-diffused silicon wafers. The pyrocatechol etchant used does not attack the boron-doped silicon so that a thin, extremely flat window results with the thickness $(1500-7500 \AA)$ determined by the depth of the boron diffusion. The crystal was mounted in a goniometer which has two orthogonal angular degrees of freedom in addition to vertical motion. The angles could be varied with $0.010^{\circ}$ accuracy. Pressure in the chamber was maintained at $10^{-7}$ Torr to reduce carbon buildup on the crystal.

The two-dimensional position-sensitive detector, which was produced at Aarhus University, ${ }^{3}$ is a solid-state silicon detector with ion-implanted resistive strips on the front and back surfaces mounted at right angles to each other. The energy and spatial resolutions are $100.0 \mathrm{keV}$ and $0.1 \mathrm{~mm}$, respectively, with nonlinearity less than $5 \%$. The detector was placed $84.8 \mathrm{~cm}$ behind the crystal. The resulting angular resolution of the system including beam divergence was $0.02^{\circ}$. A retractable screen was placed in front of the detector to allow visual observation of the transmission patterns for initial alignment of the crystal. The experimental setup is shown in Fig. 3.

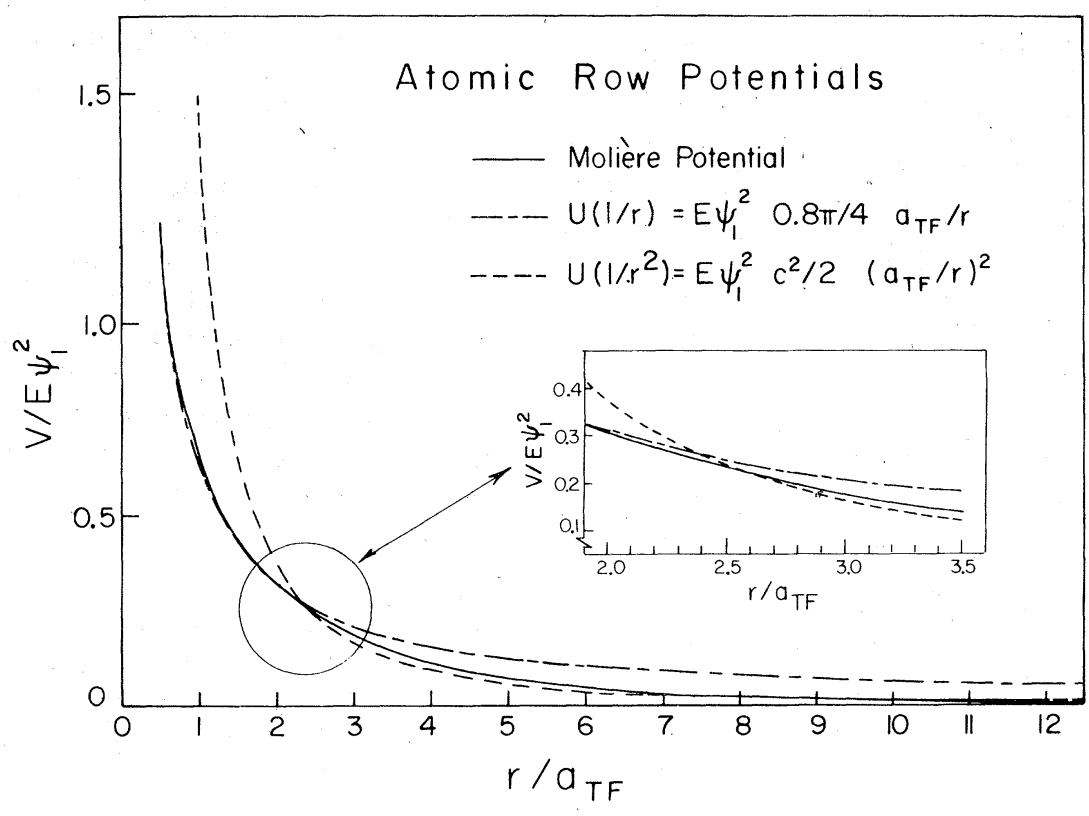

FIG. 2. Comparison of phenomenological $U(a / r)$ and $U\left(b / r^{2}\right)$ potentials with the Molière approximation to the Thomas-Fermi potential. The Molière approximation is $E \psi_{1}{ }^{2}\left[0.1 \mathrm{~K}_{0}\right.$ $\times\left(6 a_{\mathrm{TF}} / r\right)+0.55 \mathrm{~K}_{0}\left(1.2 a_{\mathrm{TF}} /\right.$ $\left.r)+0.35 \mathrm{~K}_{0}\left(3 a_{\mathrm{TF}} / r\right)\right]$. 


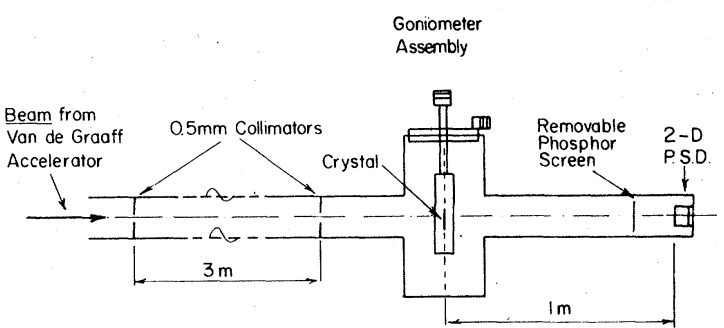

FIG. 3. Experimental setup. Beam is tightly collimated before striking the crystal, which is mounted in a precision goniometer. All transmitted particles are collected in the $2 \mathrm{D}$ position-sensitive detector. $\mathrm{x}$ - and $y$-position pulses are analyzed and collected in a twoparameter multichannel analyzer.

The pulse height $H_{p}$ at a detector contact is determined by the following relation:

$$
H_{p}=E\left[\left(x-x_{0}\right)^{2}+\left(y-y_{0}\right)^{2}\right]^{1 / 2} / L,
$$

where $E$ is the ion energy, $(x, y)$ is the ion's point of contact with the detector, $\left\{x_{0}, y_{0}\right\}$ are the con-. tact coordinates $(0,0),(0, L),(L, 0)$, and $(L, L)$, with $L$ the distance of the resistive layer between contacts. The total energy deposited was determined by summing the charge signal from both contacts, and one impact position determination could be made by dividing this energy-proportional pulse into the signal from either contact. Since the resistive layers on the front and back of the $0.5-\mathrm{mm}$-thick detector are orthogonal, the $x, y$ coordinates of the particle impact, as well as its energy, could be determined. Position and pulse height information for each event were stored in a two-parameter multichannel analyzer configured to $64-x$ channels by $64-y$ channels. The spectra were stored on magnetic tape for detailed computer analysis at a later time.

Three crystals, 1900,2500 , and $7500 \AA$ thick were studied. Scans of $\psi$ from $0^{\circ}$ (alignment with $\langle 110\rangle$ axis) to $\psi \simeq \psi_{1}$, were performed with each crystal. In addition, some variations in the azimuthal $(\phi)$ direction were investigated using the $1900 \AA$ A crystal.

\section{RESULTS}

In these transmission experiments, the data fell naturally into one of two regimes. The first is when $0<\psi \ll \psi_{1}$. In this case, particles can be properly or hyperchanneled. ${ }^{8}$ We can view this as particles trapped within a single potential minimum (Fig. 4). Analysis of this special case is outside the scope of the present report and will be presented in a future paper.

The second regime, and the one with which this paper is concerned, occurs when the particles

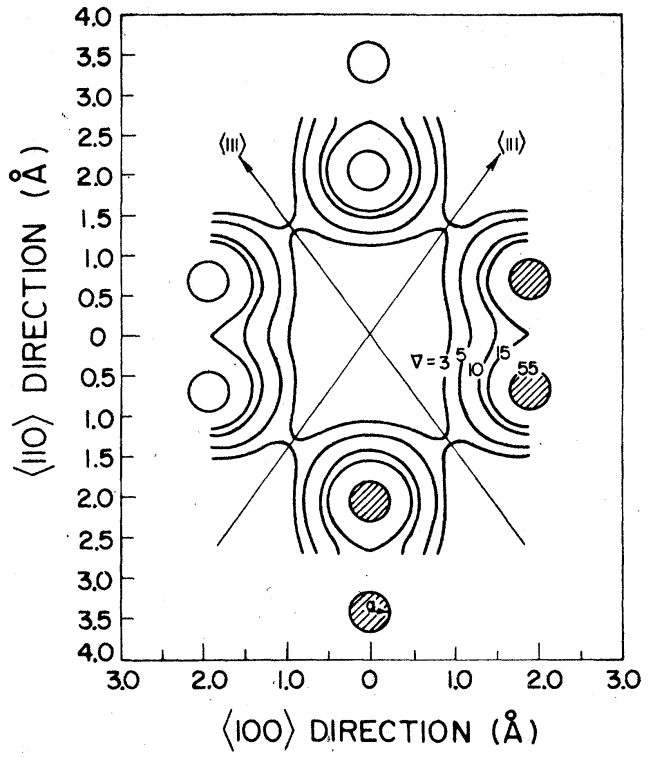

FIG. 4. Average potential contours for protons along the $\langle 110\rangle$ axial direction of silicon.

acquire enough transverse energy so that they are no longer confined to one particular axial channel, but may wander from channel to channel. This region, which is associated with most of the phase space for channeling, starts at approximately $\frac{1}{5} \psi_{1}$. In this regime of large transverse energy, the data have the following characteristics:

(i) A ringlike distribution (a doughnut) with diameter $2 D \psi$, where $D$ is the distance from the crystal to the detector.

(ii) The distribution around the doughnut is nonuniform with the highest intensity of transmitted particles generally being observed in the incident beam direction. The intensity in the azimuthal direction is also modulated by a series of minima in directions correlated with low-index-planar directions in the crystal.

(iii) The spreading of the radial distribution is found to depend on the azimuthal direction.

A typical distribution is shown in Fig. 5(a). These data were analyzed by "unfolding" the doughnuts to yield an azimuthal distribution. The procedure is described below. In addition, the radial distributions of the doughnuts in the beam direction, and at an azimuthal angle of $180^{\circ}$ to the beam direction, were also investigated.

\section{Azimuthal distributions}

The first step in the analysis of the data was to calibrate the goniometer, that is, to determine which goniometer setting corresponded to $\psi=0^{\circ}$. The diameter $W$ of the doughnut on the detector is $W=2 D\left(\psi-\psi_{0}\right)$, where $D$ is the crystal-detector dis- 
$1900 \AA$ Crystal

$$
\psi=.327^{\circ}
$$

(a)
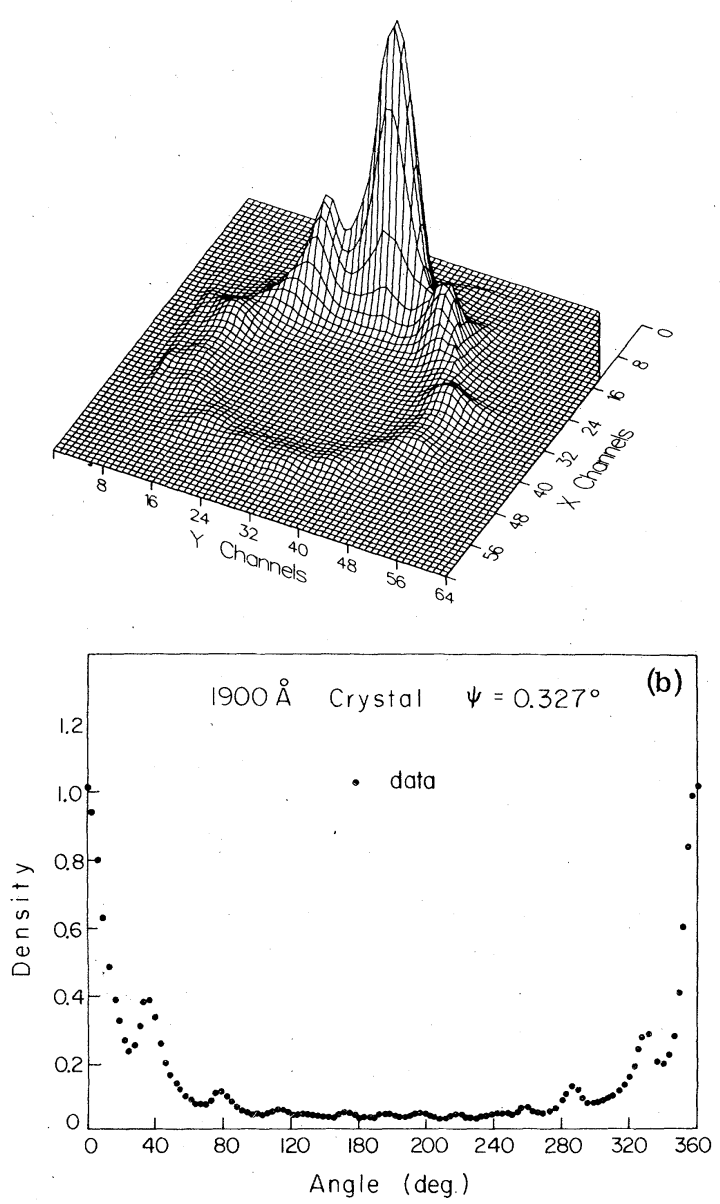

FIG. 5. (a) Doughnut formed with $\psi=0.327^{\circ}$ from $\langle 110\rangle$ axis. Crystal thickness, $1900 \AA$. (b) Azimuthal distribution of same.

tance, $\psi$ is the tilt angle measured on the goniometer, and $\psi_{0}$ is the initial tilt angle. The linear relationship illustrated in Fig. 6 between $W$ and $\psi$ was established by measurements at different values of $\psi$; extrapolation to $W=0$ then gives the goniometer setting corresponding to $\psi=\psi_{0}$.

Unfolding of the doughnuts was accomplished by dividing them into $1203^{\circ}$ wedges centered on the axis projection and integrating the counts between $d_{\min }$ and $d_{\max }$ (Fig. 7). The result was a curve of particle density versus azimuthal angle $\theta$. The curves were normalized to unit area and corrected for detector nonlinearity.

The results for the typical distributions in the two angular regions discussed above are shown in Figs. 5 and 8. Figure 8 corresponds to the special case of very small transverse energy

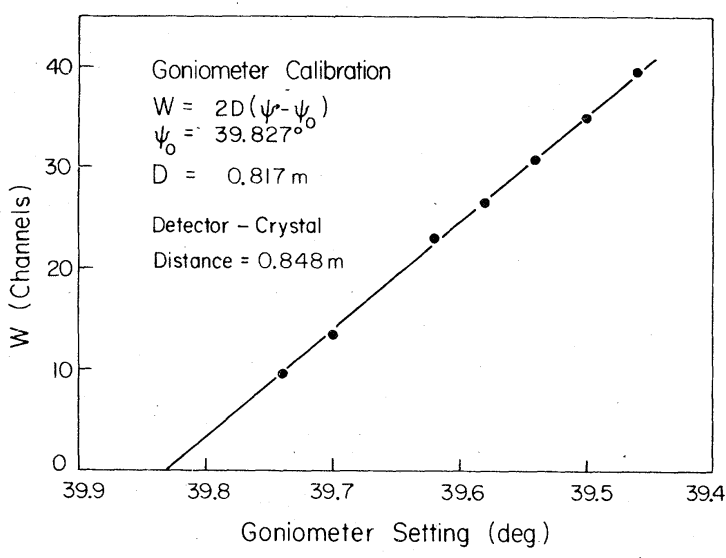

FIG. 6. Goniometer calibration $W=2 D\left(\psi-\psi_{0}\right)$ linear relation is apparent. Extrapolation to $W=0$ gives goniometer setting corresponding to $\psi=\psi_{0}$.

noted previously. This special case is characterized by an absence of an intensity maxima in the initial beam direction $(\theta=0)$ and will be discussed in detail in a subsequent paper. The more general behavior is typified by the distribution seen in Fig. 5. The theoretical distributions $A_{1 / r}$ and $A_{1 / r^{2}}$ previously discussed are tabulated and compared

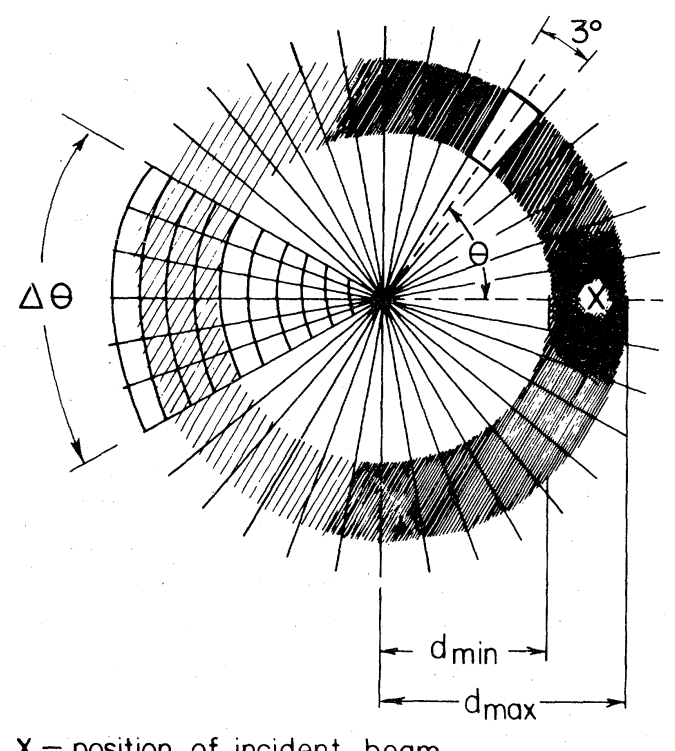

$X$ - position of incident beam

FIG. 7. Data analysis: Method for obtaining azimuthal distribution. Doughnut is divided into curvilinear boxes with angular width $3^{\circ}$ and length $d_{\max }-d_{\min }$. Counts are integrated within each box and then the distribution is normalized to unity. The radial distribution is calculated by dividing a wedge of angular width $\Delta \theta$ with equally spaced arcs. The spacing between arcs is 0.5 channels. The counts within each curvilinear box is integrated, and the distribution is normalized by dividing by the maximum numbers of counts. Therefore the highest point in any given radial distribution is 1 . 

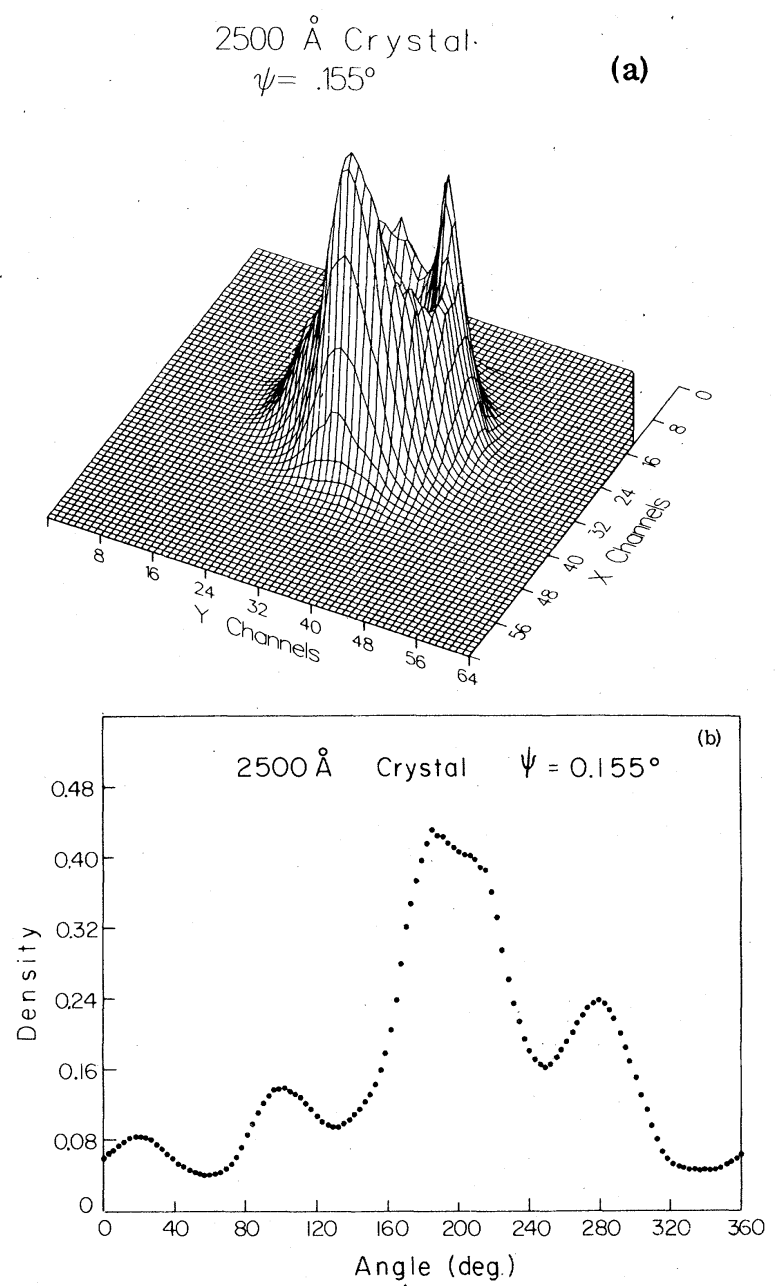

FIG. 8. (a) Doughnut formed with $\psi=0.155^{\circ}$ from $\langle 110\rangle$ axis. Crystal thicknesss, $2900 \AA$. (b) Unfolded doughnut (azimuthal distribution) $\psi=0.155$.

with the measurements for two values of the transverse energy in Figs. 9-14. In interpreting the results, the following correspondence should be noted. Small changes in $\theta$ (the angle between the incident and emergent transverse momentum directions) tend to result from small deflections of the projectile while passing through the crystal. This is the case of large impact parameter and in this limit it is expected that the $U\left(1 / r^{2}\right)$ potential will be most valid. Large changes in $\theta$ are the result of close collisions with the strings. In this regime the $U(1 / r)$ potential becomes more applicable.

1900-Å crystal

Figure 9 shows the unfolded doughnut for $\psi$ $=0.207^{\circ}=0.58 \psi_{1}$. We can see that at small values

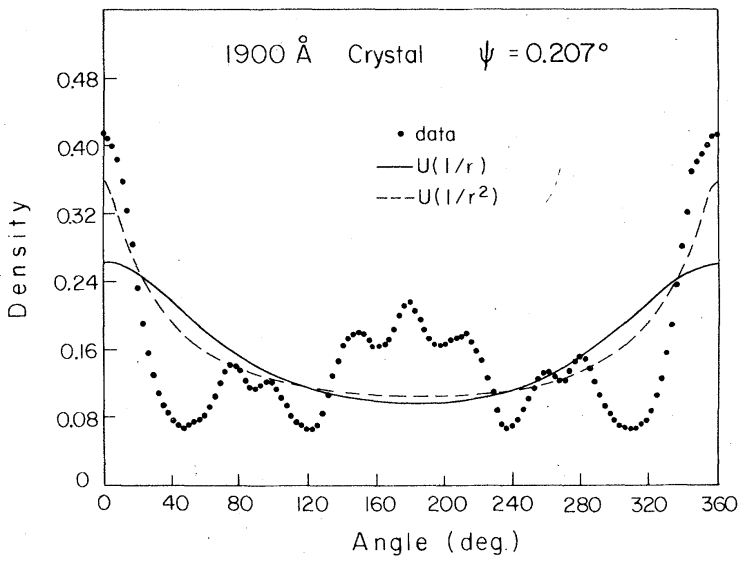

FIG. 9. Comparison of theoretical distributions with data using $U(1 / r)$ and $U\left(1 / r^{2}\right)$ potentials. Thickness is $1900 \AA$ and $\psi=0.207^{\circ}$ from $\langle 110\rangle$ axis.

of $\theta$ the $A_{1 / r^{2}}$ distribution rises faster and gives better agreement. At large $\theta$ both predictions are similar and for the overall envelope of the distribution give reasonable results. However, as $\psi$ approaches the critical angle, the flux in the beam direction rises rapidly. The distribution for $\psi$ $=0.327^{\circ}=0.99 \psi_{1}$ is shown in Fig. 10. In this case, neither potential predicts the behavior in the region of $\theta=0^{\circ}$, that is, in the beam direction. For large $\theta, A_{1 / r}$ agrees quite well, while $A_{1 / r^{2}}$ is too high. The $A_{1 / r^{2}}$ distribution is a rapidly varying function of the dimensionless parameter $T$, which is related to the path length or traversal time of a particle through a crystal and to the strength of the potential. One can see that a change of Lindhard's constant $C$ from $\sqrt{3}$ to 0.91 causes a steep rise in the region of the incident beam (i.e., $\theta \sim 0^{\circ}$ ) and depresses the distribution for large $\theta$, resulting in a reasonably good fit to the data.

A quantitative measure of the accuracy of the

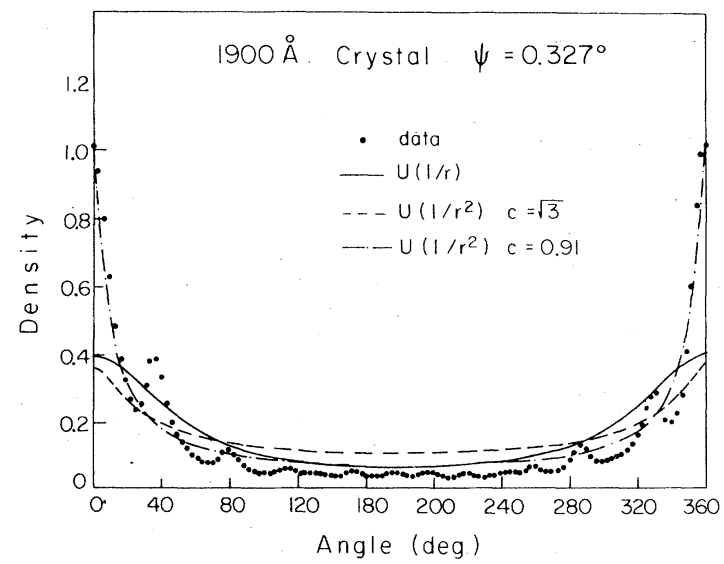

FIG. 10. Azimuthal distributions for $\psi=0.327^{\circ}$ in 1900 - $\AA$ crystal. Note $c=0.91$ yields good fit. 


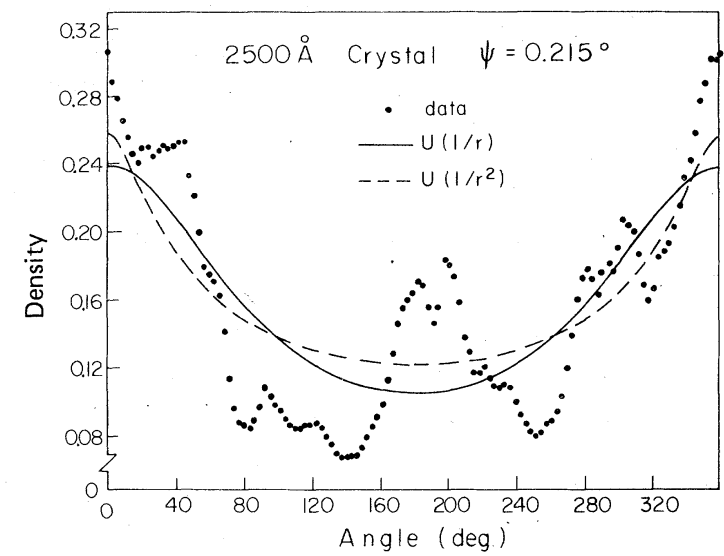

FIG. 11. Comparison of results for $2500-\AA$ crystal. $\psi=0.215^{\circ}$.

fit can be made by calculating the average projected momentum defined as

$$
\left\langle p_{\perp}\right\rangle=\int_{0}^{2 \pi} A(\theta, T) \cos (\theta) d \theta
$$

For the $A_{1 / r}$ distribution, this is simply $e^{-T_{1}}$, where $T_{1}$ is the dimensionless thickness defined in Table I. For the $A_{1 / r^{2}}$ distribution, $\left\langle p_{\perp}\right\rangle$ can be found by numerical integrations; $\left\langle p_{\perp}\right\rangle_{\text {expt }}$ is an easily tabulated quantity. The results of this analysis are presented in Table II. It is clear that none of the theoretical $\left\langle p_{\perp}\right\rangle$ 's agree well with the measured quantities. The calculated values using the $U(1 / r)$ potential vary too slowly, and the $U\left(1 / r^{2}\right)$ potential predicts no variation at all. Even when one uses the modified $C$ value, which was chosen to minimize $\chi^{2}$ in the regions $\theta=0^{\circ}$ $\pm 15^{\circ}$ and $\theta=180^{\circ} \pm 30^{\circ}$, the calculated $\left\langle p_{\perp}\right\rangle$ again varies too slowly. It is obvious that the modulations in the measured distributions caused by planar blocking effects are responsible for most of the discrepancy. Additional contributions to this quantitative lack of agreement are discussed

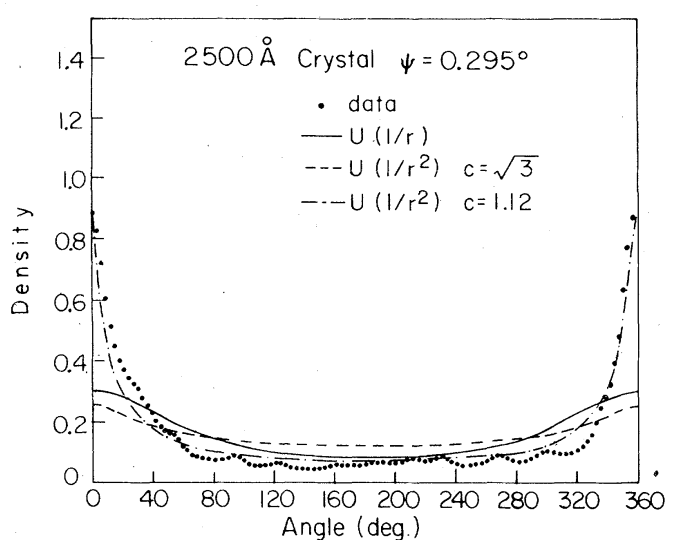

FIG. 12. Azimuthal distributions from $2500-\AA$ crystal near critical angle $\psi=0.295^{\circ}$. The envelope of the distribution is well represented using the $U\left(1 / r^{2}\right)$ potential, providing that $c$ is set equal to 1.12 .

below.

\section{0- $\AA$ crystal}

The results for both the intermediate and large values of $\psi$ are quite similar for this slightly thicker crystal. Figure 11 shows the distribution for $\psi=0.215^{\circ}=0.66 \psi_{1}$. One notes the reasonable agreement with either potential. However, near the critical angle (Fig. 12) with $\psi=0.295^{\circ}=0.9 \psi_{1}$ we again note that $A_{1 / r^{2}}$ result agrees only if we change $C$ from $\sqrt{3}$ to 1.12. Also, $A_{1 / r}$ is in agreement with the data in the region at large $\theta$ where it is expected to be most applicable.

\section{0- $\AA$ crystal}

In previous measurements, the crystal was oriented so that increasing $\psi$ tilted it into a random direction. This is defined to be approximately $15^{\circ}$ from the $\{100\}$ plane. However, in this series of experiments, the tilt was into the $\{100\}$ plane so that when $\psi \simeq \psi_{1}$ channeling in the plane became

TABLE II. Average projected momenta.

\begin{tabular}{ccccccc}
\hline \hline $\begin{array}{c}\text { Thickness } \\
(\AA)\end{array}$ & $\psi$ & $\begin{array}{c}\text { Best-fit }^{\mathrm{a}} \\
\mathrm{C}\end{array}$ & $\left\langle p_{\perp}\right\rangle_{1 / r}$ & $\left\langle p_{\perp}\right\rangle_{1 / r^{2}}{ }^{\mathrm{b}}$ & $\left\langle p_{\perp}\right\rangle_{1 / r^{2}}{ }^{\mathrm{c}}$ & $\left\langle p_{\perp}\right\rangle_{\text {expt }}$ \\
\hline 1900 & $0.207^{\circ}$ & 1.732 & 0.281 & 0.249 & 0.249 & 0.109 \\
1900 & $0.247^{\circ}$ & 1.458 & 0.346 & 0.249 & 0.311 & 0.249 \\
1900 & $0.287^{\circ}$ & 1.139 & 0.401 & 0.249 & 0.403 & 0.467 \\
1900 & $0.327^{\circ}$ & 0.910 & 0.449 & 0.249 & 0.486 & 0.604 \\
2500 & $0.215^{\circ}$ & 1.732 & 0.200 & 0.160 & 0.160 & 0.267 \\
2500 & $0.255^{\circ}$ & 1.220 & 0.258 & 0.160 & 0.276 & 0.414 \\
2500 & $0.295^{\circ}$ & 1.120 & 0.310 & 0.160 & 0.307 & 0.534 \\
\hline \hline
\end{tabular}

${ }^{\mathrm{a}}$ For envelope of distribution derived from $U\left(1 / r^{2}\right)$ potential.

${ }^{\mathrm{b}}$ Using $C=\sqrt{3}$.

${ }^{c}$ Using best-fit $C$ values shown in column 3 . 


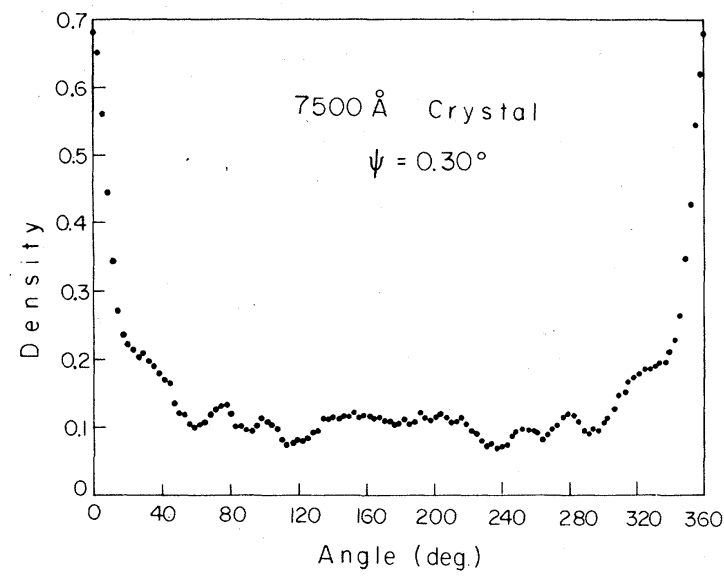

FIG. 13. Azimuthal distributions near critical angle in $7500-\AA$ crystal. The very large flux near $\theta=0^{\circ}$ is caused by channeling in $\langle 100\rangle$ plane.

important. Consequently, the large flux in the forward direction for $\psi=0.3^{\circ}=0.91 \psi_{1}$ is the result of channeling in the $\langle 100\rangle$ plane (Fig. 13). However, at $\psi=0.2^{\circ}=0.66 \psi_{1}$, the protons are not planar channeled and the distribution can be compared with the theoretical predictions. In passing through this thick crystal, the protons made a sufficient number of collisions with the strings so that statistical equilibrium was achieved. Thus both theoretical distributions are flat, as are the data, with the exception of the large blocking dips (Fig. 14).

\section{DISCUSSION}

In Sec. IV, measurements of azimuthal doughnut distributions and average projected momenta as a function of crystal thickness and transverse energy have been presented. It is also seen that the ran-

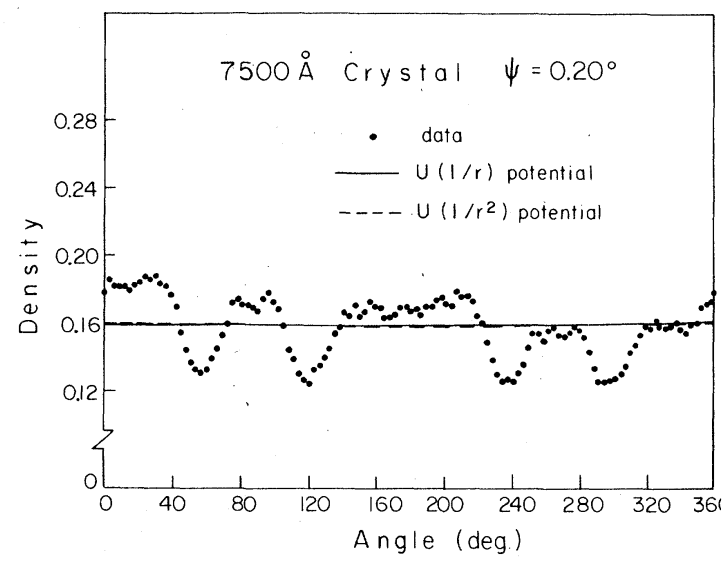

FIG. 14. 7500- ̊ crystal azimuthal distributions. Note that both $U(1 / r)$ and $U\left(1 / r^{2}\right)$ potentials result in uniform distributions. dom string approximation predicts envelopes for these angular distributions that are not wildly in conflict with the data. Nevertheless, it is clear that detailed agreement between experiment and this theory have not been achieved. First there are the obvious planar features that cause deviations from the smooth angular yields predicted by the theory. It is clear that a calculation based upon the assumption of a random distribution of strings in the transverse plane cannot account for these features. An ad hoc way of dealing with these planar blocking dips is to consider the planar features predicted by a simple two-string model. A particle emitted from the first string will be steered away from the direction of the second. This "shadow behind the string" effect can be estimated from the potential. ${ }^{9}$ When the Lindhard standard interatomic potential [ Eq. (2)] is used together with the approximation of smallangle scattering, the angle $\theta_{\min }$ for blocking in the transverse plane is found to be

$$
\theta_{\min }=\frac{3}{2}\left(\frac{\pi}{2} \frac{E \psi_{1}^{2} C^{2} a_{T F}^{2}}{E_{\perp} d_{s}^{2}}\right)^{1 / 3},
$$

where $E_{\perp}$ is the transverse energy and $d_{s}$ is the distance bet ween strings. In Figs. 13 and 14, the deep dips are caused by the $\{111\}$ planes. The predicted values of $\theta_{\min }$ are $32.1^{\circ}$ and $28.1^{\circ}$ for $\psi=0.2^{\circ}$ and $0.3^{\circ}$, respectively. The experimental values are $24^{\circ}$ for $\psi=0.2^{\circ}$ and $21^{\circ}$ for $\psi=0.3^{\circ}$. These results are quite reasonable considering the fact that the calculation is based only on a two-string smallangle scattering model.

The problem of explaining the deviation between the random string approximation and the measured average projected momenta and envelope of the angular-distribution function is more involved. At first glance, there are several possible explanations. They are (a) neither the $1 / r$ nor $1 / r^{2}$ potential satisfactorily represents the string potential, (b) correlation effects are not directly included in the above two-string calculation, or (c) inhomogenieties in the crystal thickness.

We discuss the effect of potential first. The data for the large azimuthal angle behavior for large transverse energies (Figs. 10 and 12) are in good agreement with the RSA. This is quite reasonable since for such thin crystals those particles that scatter to large angles clearly have had a hard string scattering with a small distance of closest approach. The scattering angles are therefore dominated by the potential at small distances from the string and from Fig. 2 the $1 / r$ potential clearly represents this well. Still staying with large transverse energies but now going to small azimuthal angles, the data can be much better repre- 
sented by the $1 / r^{2}$ potential than the $1 / r$. However, in order to get a good fit it is necessary to modify the standard value of $C$ to $\sim 1.0$. No analogous simple adjustment of the $1 / \gamma$ potential yields good agreement in this region.

Before interpreting this result as a significant comment on the interatomic potential we must consider smaller transverse energy data (Figs. 9 and 11). Here the smallest impact parameter events are able to penetrate the region where the $1 / r$ potential is a satisfactory representation (Fig. 2). However, most of the collisions occur in the $1 / r^{2}$ region. Clearly from Figs. 9 and 11 the $1 / r^{2}$ fit seems satisfactory at small angles but only for an unmodified value of $C$ (i.e., $C=\sqrt{3}$ ). Using the previously discussed value of $C \simeq 1.0$ leads to a very poor description at small azimuthal angles. It is clearly unsatisfactory (and inconsistent) to represent the $1 / r^{2}$ potential so differently for different transverse energies. (Table II contains values of $C$ which produce best fits to the envelope function for different crystal thicknesses and tilts.)

The origin of this dilemma may well be in correlation effects associated with the nonrandom distribution of strings in the transverse plane. Recent experiments on multiple scattering in polycrystalline material have shown that even in the absence of channeling, crystalline effects can cause large deviations between predicted and measured angular distributions. ${ }^{10}$ Correlation effects also influence the multiple scattering from polyatomic gases. ${ }^{11}$. Whether or not this is the case in the present experiments is not clear but in view of this possibility we refrain from interpreting our result in terms of modified potential functions. Work is currently underway to clarify this question both within the framework of the RSA and also by Monte Carlo calculations. ${ }^{12}$

\section{Radial distributions}

The radial distributions were obtained by integrating the counts within some wedge of finite angular width $\Delta \theta$. The wedge was first divided with equally-spaced arcs. The counts within each curvilinear box were then summed (Fig. 7). The curves were normalized by dividing the number of counts in each box by the maximum number of counts.

Although we have no quantitative theoretical predictions for these radial distributions, it is instructive to consider the qualitative features. Figures 15 and 16 show the radial distributions for different incident transverse energies $E_{\perp}\left(E_{\perp}=E \psi^{2}\right)$ in the region of the doughnut corresponding to the incident beam direction $\theta=0^{\circ}$, and for particles that have undergone a large change in the direction

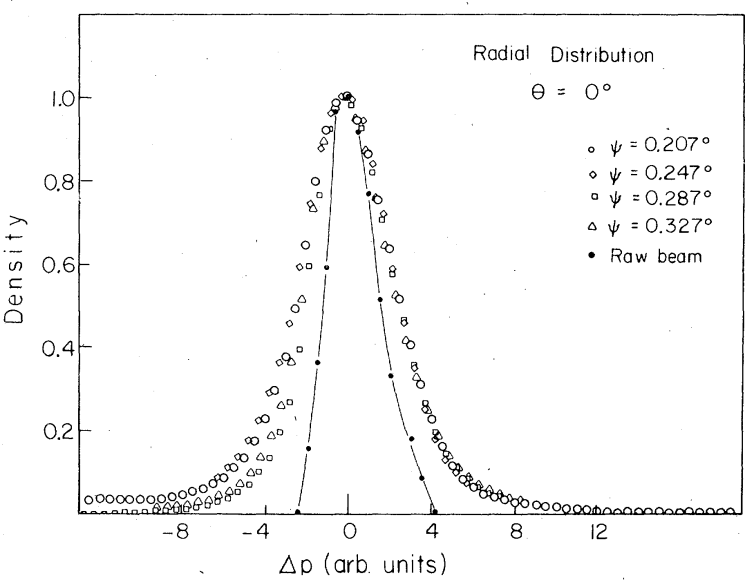

FIG. 15. Radial distribution in the beam direction $\theta$ $=0^{\circ}$ for various $\psi$ 's.

of the transverse momentum vector $\theta=180^{\circ}$, respectively. The distance scale is proportional to the magnitude of the final transverse momentum. The shape of the curves is determined by three independent events. When a particle enters the crystal, its transverse energy increases by an amount $U\left(\boldsymbol{r}_{i}\right)$ over its initial value $E \psi^{2}$, where $U\left(r_{i}\right)$ is the value of the potential at the point of entry $r_{i}$. Likewise, as it leaves the crystal its energy decreases by $U\left(\boldsymbol{r}_{f}\right)$. Finally, if it undergoes inelastic transverse (multiple) scattering inside the crystal, its transverse energy changes by an additional amount. Thus the final distribution of radial transverse momentum is determined by the distribution of $\boldsymbol{r}_{\boldsymbol{i}}$ and $\boldsymbol{r}_{\boldsymbol{f}}$ values and any effects of inelastic transverse multiple scattering. Furthermore, experimental problems such as finite beam divergence and multiple scattering in

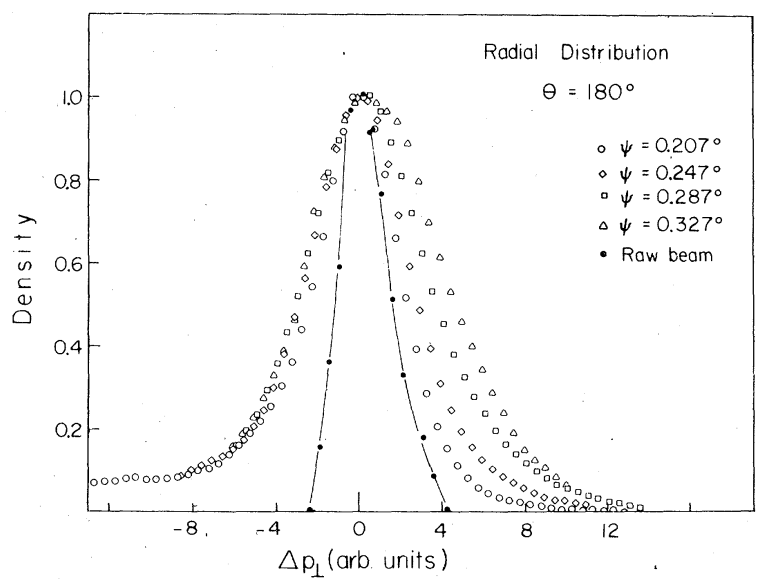

FIG. 16. Radial distribution for particles which have undergone a reversal of direction of the transverse momentum vector by single scattering from an atomic row (string) $\theta=180^{\circ}$. 
oxide layers can contribute to the radial spread.

For thin crystals (i.e., far from equilibrium) those particles which exit the crystal with $p_{\perp}$ in the same direction as the incident beam direction (i.e., $\theta \sim 0^{\circ}$ ) have in general stayed away from close interactions with atomic rows. They have therefore avoided the strong inelastic effects associated with multiple scattering in high electron density regions and off of thermal vibrations of the strings. We can see from Fig. 15 that the corresponding radial distributions are symmetric and do not change shape for different values of $\psi$. (Note that in this figure the horizontal scales have been shifted so that peak positions line up, i.e., the initial transverse momentum has been subtracted so that $\Delta p=0$ corresponds to exiting transverse momentum $p \psi$.) In this case, the magnitude of the width of the radial distribution, as well as the invariance of this quantity towards changes in $\psi$, is completely consistent with this distribution being dominated by beam divergence and oxidelayer multiple scattering.

On the other hand, for particles exiting at $\theta \sim 180^{\circ}$, a stronger interaction with (i.e., closer approach to) strings will result in increased contribution to this width from multiple scattering on thermal vibrations. Thus the radial distribution is clearly seen to be broadened for particles in the region of $\theta=180^{\circ}$ (Fig. 16). This effect increases for increasing $\psi$ as is to be expected since the distance of closest approach to the strings is decreasing. These results represent the first direct measurement of the impact parameter dependence of inelastic transverse scattering (commonly called multiple scattering). Such scattering is the origin of dechanneling effects and it is hoped that additional measurements of the type shown in Figs. 15 and 16 will provide quantitative understanding of such effects.

\section{ACKNOWLEDGMENTS}

This work was supported in part by the NSF, and by the Division of Basic Energy Sciences, Department of Energy, under Contract No. EY-76-C-020016 .

\footnotetext{
*Present address: Physics Dept., Brookhaven National Laboratory, Upton, N. Y. 11973.

$\dagger$ Present address: Dept. of Physics, State University of New York, Albany, N. Y. 12203.

${ }^{1}$ D. D. Armstrong, W. M. Gibson, A. Goland, J. A. Golovchenko, R. A. Levesque, R. L. Meek, and H. E. Wegner, Radiat. Eff. 12, 143 (1972).

${ }^{2}$ When the transverse energy of an impinging charged particle is less than the continuum potential energy of the crystal rows or planes, the motion is governed by the potential, and directional effects due to the crystal's symmetry are observed. This governed motion is known as channeling. The critical or characteristic angle $\psi_{1}$ is defined as $\left(2 Z_{1} Z_{2} e^{2} / E d\right)^{1 / 2}$, where $Z_{1} e$ and $Z_{2} e$ are the projectile and target charge respectively, $E$ is the incident beam energy, and $d$ is the interatomic spacing.

${ }^{3}$ The two-dimensional position-sensitive detector was fabricated by Erik Laegsgaard of Aarhus University.
}

${ }^{4}$ The samples were prepared by Mary Lou Shombert, Bell Laboratories.

${ }^{5}$ J. A. Golovchenko, Phys. Rev. B 13, 4672 (1976).

${ }^{6} \mathrm{~J}$. Lindhard, K. Dan. Vidensk. Selsk. Mat.-Fys. Medd. 34, 14 (1965).

${ }^{7}$ G. Molière, Z. Naturforsch A $\underline{2}, 133$ (1947).

${ }^{8}$ B. R. Appleton, J. H. Barrett, J. S. Noggle, and C. D. Moak, Radiat. Eff. 13, 171 (1972); B. R. Appleton, C. D. Moak, J. S. Noggle, and J. H. Barrett, Phys. Rev. Lett. 28, 1307 (1972).

${ }^{9} \mathrm{~J}$. A. Golovchenko, Ph.D. thesis (Rensselaer Polytechnic Institute, 1972) (unpublished).

${ }^{10} \mathrm{H}$. H. Andersen, J. Bøttiger, H. Knudsen, P. Møller Petersen, and J. Wohlenberg, Phys. Rev. A 10,1568 (1974).

${ }^{11}$ P. Sigmund, K. Dan. Vidensk. Selsk. Mat.-Fys. Medd. 39, 14 (1977).

${ }^{12}$ J. Ellison, S. T. Chui and W. M. Gibson, Bull. Am. Phys. Soc. 22, 327 (1977); (unpublished). 